\title{
Ultrasonography helps emergency physician identify the best lumbar puncture site under the conus medullaris
}

\author{
Line Dussourd, Batistin Martinon, Clara Candille, Carole Paquier, Claire Wintenberger, Perrine Dumanoir,
} Anais Plazanet, Damien Viglino and Maxime Maignan * (1)

\begin{abstract}
Background: Ultrasonography - assisted lumbar puncture helps physicians identify traditional anatomical landmarks. However, it could help to overcome the anatomical dogmas and thus identify the best interspinous space under the medullary cone.

Methods: Traditional anatomical landmarks were reported on a tracing paper in patients with an indication for lumbar puncture. Then, ultrasonography was used to locate the optimal interspinous level defined as the widest subarachnoid space located below the conus medullaris. Primary endpoint was the distance between traditional and ultrasound landmarks.

Results: Fifty-seven patients were included. Seven emergency physicians practiced the procedure. The median absolute distance between traditional anatomical landmarks and ultrasound marking was 32 [interquartile (IQR) 27 - 37] mm. The inter-spinous space identified in the two procedures was different in $68 \%$ of the cases.

Conclusions: Ultrasound not only allows us to better identify anatomical structures before lumbar puncture, but it also allows us to choose a site of puncture different from recommendations.
\end{abstract}

Keywords: Ultrasonography, Lumbar puncture, Emergency

\section{Dear Editor}

Lumbar puncture is a common procedure in emergency departments, with 0.8 procedures per 100 admissions [1]. The failure rate of this procedure is close to $10 \%$ and complications such as postdural puncture headache are not uncommon [2]. There is increasing evidence for the benefits of ultrasound-assisted lumbar puncture, particularly in children and infants [2, 3]. The main benefit of ultrasound localization of puncture site is the easy identification of traditional anatomical landmarks $[4,5]$. In fact, anatomical assessment of intervertebral space level for lumbar puncture is misleading in more than $36 \%$ [6]. The rates of failure and complications after lumbar puncture are thus diminished due to the anatomical localization capabilities of ultrasonography [2]. However, we believe that the main interest of

* Correspondence: mmaignan@chu-grenoble.fr Emergency Department, CHU Grenoble Alpes, CS 10217, 38043 Grenoble, cedex 9, France

(c) The Author(s). 2017 Open Access This article is distributed under the terms of the Creative Commons Attribution 4.0 International License (http://creativecommons.org/licenses/by/4.0/, which permits unrestricted use, distribution, and reproduction in any medium, provided you give appropriate credit to the original author(s) and the source, provide a link to the Creative Commons license, and indicate if changes were made. The Creative Commons Public Domain Dedication waiver (http://creativecommons.org/publicdomain/zero/1.0/) applies to the data made available in this article, unless otherwise stated. ultrasonography studies lies in a more comprehensive anatomical approach. Instead of simply locating the interspinous space L3-L4 or L4-L5, ultrasound allows the location of the optimal interspinous level below the conus medullaris. This approach has been recently tested in infants [3]. We have also used this approach in adults to investigate the impact of ultrasonography on the choice of the lumbar puncture site.

\section{Methods}

Patients gave their written informed consent and the study was approved by the local ethic committee. We selected patients presenting to the emergency department with an indication for lumbar puncture because of febrile or sudden headache. Whether or not the lumbar puncture was performed was left to the discretion of the emergency physician in charge of the patient. The physician performing the study was not involved in patient care. The physician in charge of the patient was not kept 
informed of the results of the patient. Emergency physicians performing the procedures had different level of experience ( 2 residents, 1 chief resident, 3 attending physicians and 1 associate professor). They were all accustomed to using ultrasound in daily medical practice (FAST echo, basic cardiac ultrasonography etc.). Before participating in the study, they were specifically trained by an anesthesiologist to identify interspinous levels. This training did not exceed $30 \mathrm{~min}$.

Briefly, the procedure for the study was performed with a Vivid S70 device (GE healtcare, Little Chalfont, UK). There was no supervision or validation of the anatomical and ultrasound procedures. Patients were randomized to have a location of the puncture site in a sitting position or in lateral decubitus to avoid any bias linked to patients' position. Once in position, a tracing paper was placed on the back of the patient to make four marks with a surgical marking pen. This process made it possible to reposition the tracing paper in a reproducible way. An emergency physician carried out the traditional anatomical landmark of the lumbar puncture site, and reported its location only on the tracing paper. He then used ultrasound to locate the optimal interspinous level defined as the widest subarachnoid space located below the conus medullaris. The location identified by ultrasound was reported on the tracing paper. The distance between the two marks was measured as well as the distance on the carniocaudal axis and the mediolateral axis.
Measurements were made by an independent emergency physician.

\section{Results}

We included 52 patients including 17 (33\%) men. The mean age was 45 (95\% CI $(18$ - 84)) years and body mass index was 25 (range 17 to 41 ) $\mathrm{kg} / \mathrm{m} 2$. Patients were admitted to the ED for meningeal syndrome $(n=9$, $17 \%)$, sudden headache $(n=2,4 \%)$, unusual headache $(n$ $=27,52 \%)$, and febrile headache $(n=14,27 \%) .28$ markings were made in sitting position and 24 in the lateral decubitus. The median absolute distance between traditional anatomical landmarks and ultrasound marking was 32 [interquartile (IQR) 27 - 37] $\mathrm{mm}$. The ultrasound mark was almost systematically below the anatomical mark which corresponded to a distance on the craniocaudal axis of 29 [IQR $24-34] \mathrm{mm}$ (Fig. 1). The distance on the mediolateral axis was 8 [IQR 6 - 10] $\mathrm{mm}$. The rate of change of interspinous space was $68 \%$ in the sitting position group and $67 \%$ in the lateral decubitus group. There was no significant correlation between body mass index (BMI) and the distance between traditional anatomical landmarks and ultrasound marking (Spearman correlation coefficient: 0.006, $p=0.97$ ). Obese patients with a BMI of greater than $30 \mathrm{~kg} / \mathrm{m}^{2}$ tended to have a higher risk of interspinous space change (risk ratio $=1.45$ [95\% confidence interval: 1.06 1.99]; $p=0.14$ Fisher exact test).

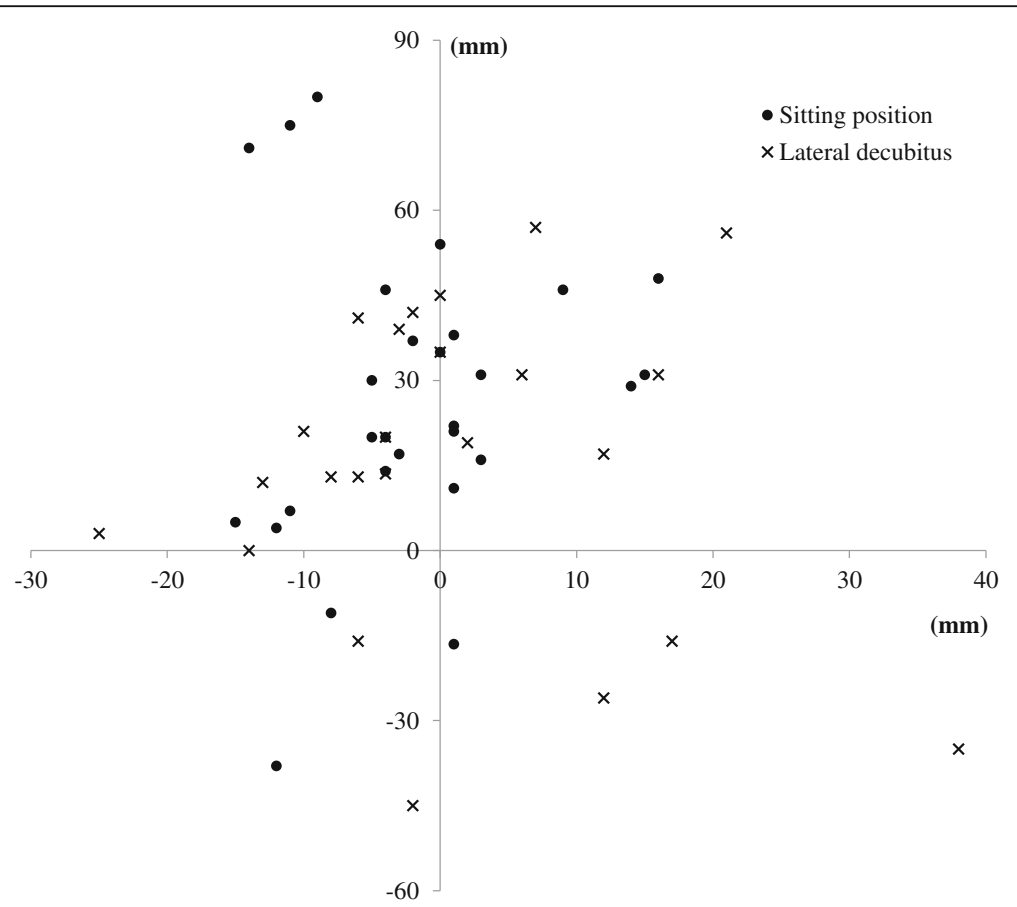

Fig. 1 Craniocaudal and mediolateral distances between ultrasound marking and traditional anatomical marking. The center point represents ultrasound marking. Cross: sitting position; Circle: lateral decubitus 


\section{Discussion}

Our results confirm the value of ultrasonography to correctly identify both anatomical landmarks (midline, spinous processes etc.) and the optimal interspinous space for lumbar puncture. Ultrasound marking was mostly located below the anatomical palpation marking, which is in line with previously published data $[7,8]$. Ultrasonography marking did not seek to identify the inter-spinous space L2-L3 or L3-L4, but the intervertebral space which seemed to be the most conducive to the introduction of a lumbar puncture needle. This choice was voluntary in order to be as close as possible to the realization of procedure in the emergency department. Interestingly, the conus medullaris ends in most cases in the intervertebral space L1-L2 but in 2 to $5 \%$ of cases it descends to below the vertebral body of L2 [9]. Furthermore, the shape of thecal sac varies in lower interspace making the success of the lumbar puncture more uncertain [10]. We thus believe that the main advantage of ultrasound marking is related to the identification of lower interspinous level than those traditionally chosen after anatomical marking. Our results suggest that the benefits of the ultrasound technique would be even greater in obese patients, which is consistent with previous reports [11]. Ultrasound marking allows a safe needle introduction to be made below the conus medullaris while allowing the catheterization of the thecal sac even in low interspinous spaces. Regarding the abundant literature advocating very explicitly the use of this technique, a randomized clinical trial comparing success rates using the two techniques in adults should now be carried. A study on the impact of ultrasonography on reducing the rate of post dural puncture headache could also be interesting.

\section{Conclusions}

Ultrasound not only allows us to better identify anatomical structures before lumbar puncture, but it also allows us to choose a better site of puncture. Regarding the abundant literature advocating very explicitly the use of this technique, a randomized clinical trial comparing success rates using the two techniques in adults should be carried. A study on the impact of ultrasonography on reducing the rate of post dural puncture headache could also be useful.

\section{Abbreviations}

IQR: Interquartile

Acknowledgements

We greatly thank Alison Foote for editing the manuscript.

\section{Availability of data and materials}

The datasets used and/or analyzed during the current study are available from the corresponding author on reasonable request.

\section{Authors' contributions}

MM had full access to all of the data in the study and takes responsibility for the integrity of the data and the accuracy of the data analysis. LD, BM, CC, DV and MM participated in data acquisition, analysis, and interpretation. DV, AP and MM drafted the manuscript. LD, BM, CC, CP, CW and PD made critical revision of the manuscript for important intellectual content. $C C, C P$, CW, PD, AP, DV and MM contributed to administrative, technical, or material support. All authors read and approved the final manuscript.

\section{Competing interests}

The authors declare that they have no competing interests.

\section{Consent for publication}

Not applicable

\section{Ethics approval and consent to participate}

Local institutional review board approved the study; all participants gave their written informed consent.

\section{Publisher's Note}

Springer Nature remains neutral with regard to jurisdictional claims in published maps and institutional affiliations.

Received: 23 March 2017 Accepted: 14 June 2017

Published online: 27 June 2017

\section{References}

1. Moisset X, Ruet A, Brochet B, Planche V, Jaffeux P, Gilleron V, et al. Who Performs Lumbar Puncture, How Many Do They Perform, How and Why? A Retrospective Study of 6,594 Cases. Eur Neurol. 2016;76:8-11.

2. Shaikh F, Brzezinski J, Alexander S, Arzola C, Carvalho JCA, Beyene J, et al. Ultrasound imaging for lumbar punctures and epidural catheterisations: systematic review and meta-analysis. BMJ. 2013;346:f1720.

3. Neal JT, Kaplan SL, Woodford AL, Desai K, Zorc JJ, Chen AE. The Effect of Bedside Ultrasonographic Skin Marking on Infant Lumbar Puncture Success: A Randomized Controlled Trial. Ann Emerg Med. 2017;69(5):610-9.

4. Ferre RM, Sweeney TW. Emergency physicians can easily obtain ultrasound images of anatomical landmarks relevant to lumbar puncture. Am J Emerg Med. 2007;25:291-6.

5. Hayes J, Borges B, Armstrong D, Srinivasan I. Accuracy of manual palpation vs ultrasound for identifying the L3-L4 intervertebral space level in children. Paediatr Anaesth. 2014:24:510-5.

6. Duniec L, Nowakowski P, Kosson D, Łazowski T. Anatomical landmarks based assessment of intravertebral space level for lumbar puncture is misleading in more than 30\%. Anaesthesiol Intensive Ther. 2013;45:1-6.

7. Broadbent CR, Maxwell WB, Ferrie R, Wilson DJ, Gawne-Cain M, Russell R. Ability of anaesthetists to identify a marked lumbar interspace. Anaesthesia. 2000:55:1122-6.

8. Schlotterbeck H, Schaeffer R, Dow WA, Touret Y, Bailey S, Diemunsch P. Ultrasonographic control of the puncture level for lumbar neuraxial block in obstetric anaesthesia. Br J Anaesth. 2008;100:230-4.

9. Whitty $\mathrm{R}$, Moore $M$, Macarthur $A$. Identification of the lumbar interspinous spaces: palpation versus ultrasound. Anesth Analg. 2008;106:538-40. table of contents.

10. Naji M, Williams M, Hourihan MD, Collis RE. Shape of the thecal sac: L3/4 interspace compared with L4/5. Anaesthesia. 2009;64:39-42.

11. Stony R. Ultrasound-assisted lumbar puncture in obese patients. Crit Care Clin. 2010;26:661-4. 